\title{
Enhancement of Aerodynamic Efficiency of Aerofoil Using Improved Blowing and Suction System
}

\author{
K. Balaji ${ }^{1,2}$, G. Jims John Wessley ${ }^{1 *}$ \\ 1 Karunya Institute of Technology and Sciences, Coimbatore, Tamilnadu, India \\ 2 Department of Aeronautical Engineering, Sanjay Ghodawat University, Kolhapur, Maharashtra, India \\ * Corresponding author's email: jims_john@karunya.edu
}

\begin{abstract}
Improved Blowing and Suction System (IBSS) is a novel concept that can be effectively implemented in future aircraft to improve the aerodynamic performance of aircraft wings. The proposed IBSS consists of a regular wing without its secondary control surfaces and with a Pump. The injection and suction system is used to create additional flow without disturbing the main flow over the aerofoil which effectively delays the boundary layer separation. The injection and suction areas are kept constant and they are located just below the maximum thickness point and suction is created close to the trailing edge. This paper presents a detailed numerical analysis of the proposed IBSS and the study shows that the stalling angle of attack is increased by $60 \%$ while the coefficient of lift is increased $37.5 \%$ compared to the baseline aerofoil. Also, the commercially used coflow jet (CFJ) method stalls at a $12^{\circ}$ angle of attack whereas with the proposed IBSS method the stall occurs at a $16^{\circ}$ angle of attack. The stalling angle is thus delayed by $33 \%$, thus increasing the aerodynamic performance of the wing as compared to the existing methods. Also, any malfunction in IBSS does not significantly affect the performance of the aircraft. The implementation of IBSS will lead to a reduction in the weight of the aircraft and the elimination of secondary control surfaces.
\end{abstract}

Keywords: co flow jet, improved blowing and suction system, aerodynamic efficiency, stalling angle, control surfaces, secondary flow.

\section{INTRODUCTION}

In recent years, researchers are working on improving the performance of aircraft wings by modifying their configuration to effectively control the boundary layer that will shift the flow separation point positively. To achieve this, active and passive control mechanisms are integrated into the wing surfaces which results in increasing the stalling angle of attack and coefficient of lift. Thus efficient control of the boundary layer separation in aircraft wings even at low speeds can be achieved. In today's aircraft, vortex generators, slats and flaps, synthetic jet methods are normally used to control the boundary layer separation over an aircraft wing. Another important method secondly the losses incurred due to the shift in the secondary flow moving away from the leading edge towards the trailing edge. Thus, for performance enhancement of aerofoils, there is a need for small and complicated additional components to achieve improved flight performance, leading to an increase in the dead-weight, accompanied by increased maintenance costs. These systems also have the possibility of failing the entire controls in case of a small malfunction in any of the components associated with it. These problems can be avoided with the use of the proposed IBSS that improves the performance as well as enables a reduction in the weight of the wing by eliminating the need for additional components.

Zha and Paxton [1] conducted a numerical investigation over a NACA 2415 aerofoil under takeoff, landing and cruise conditions. It's seen 
that the stalling angle of attack increased by 2 degrees and during operation, the angle of attack is found to have improved by $38 \%$ and lift is also is enhanced by $80 \%$. The study also shows that at a low angle of attack more lift is generated than at a higher angle of attacks due to the formation of the wake region.

Zha and Paxton [2] performed numerical analysis over a NACA 2415 aerofoil to predict the optimum location of injection and suction to obtain higher efficiency. The study revealed that to obtain more aerodynamic efficiency, the injection slot must be located close to the leading edge and the suction slot must be located close to the trailing edge. Alexis Lefebvre and Zha [3] numerical investigated a NACA 23121 aerofoil with constant Mach number 0.15 and Reynolds number $6.14 \times 10^{6}$. In this study, the influence of injection and suction location to obtain improved aerodynamic efficiency and reduced power consumption is estimated. The study suggests that the reduction in power consumption by positioning the injection location upstream is directly proportional to the angle of attack. Zha et al. [4] researched NACA 0025 aerofoil with the co-flow jet method using CFD software. It is seen from the study that, in the symmetrical aerofoil, the lift is enhanced by $220 \%$ and stall margin is improved by $153 \%$ when compared to the traditional methods.

Zha et al. [5] performed simulation on NACA 0025 aerofoil with constant Mach number and Reynolds number and different angle of attack and momentum coefficients. The study shows that the lift is improved from $113 \%$ to $220 \%$ and stall margin is enhanced from $100 \%$ to $153 \%$, while the drag value reduced from $30 \%$ to $127 \%$. Hossain et al. [6] investigated a NACA 0015 aerofoil incorporating CFJ mechanism in a subsonic wind tunnel with the input parameters as the velocity of $12 \mathrm{~m} / \mathrm{sec}$, jet momentum 0.07 and mass flow rate $0.030 \mathrm{~kg} / \mathrm{sec}$. The study revealed that the lift is improved by $82.5 \%$ and drag is reduced by $16.5 \%$ as compared to the baseline aerofoil. Lefebvre et al. [7] conducted an experimental and numerical analysis to calculate the aerodynamic performance and to estimate the energy used to operate a CFJ 6415 aerofoil. The inlet conditions for this analysis were taken as different Mach numbers and angle of attack at constant jet momentum under cruise condition. It shows that the CFJ aerofoil increased the stalling angle of attack by $120 \%$ at the cruise condition. One problem noticed during the study is that after a certain Mach number, a shock wave is created which affects the performance of the aerofoil.

Balaji and Wessley [8] conducted a wind tunnel test and showed that the stalling angle of attack improved by 5 degrees as compared to the baseline aerofoil on a NACA 6415 aerofoil. Results proved that the coefficient of lift is enhanced by $43 \%$ compared to the baseline aerofoil. However, due to the lift dependent drag, the coefficient of drag also marginally increased. Lefebvre and Zha [9] performed 3D analysis over a NACA 6415 aerofoil for different phases of flight. In this analysis, various parameters like injection suction location, location of cavity and aspect ratio at Mach number from 0.10 to 0.15 were tested. It is seen that more $\mathrm{C} \mu$ and energy are needed for takeoff and landing conditions whereas fewer $\mathrm{C} \mu$ and energy were required to achieve good aerodynamic efficiency and maximum coefficient of lift during other phases of flight. Dano et al. [10] carried out wind tunnel testing on a CFJ aerofoil to predict the turbulence influence at low and high angle of attacks. From the study, it is seen that at a high angle of attack the occurence of turbulence mixing with the main flow enhanced the performance of the aerofoil.

From the literature survey, it is seen that the CFJ method is implemented in few aerofoils for enhancing the performance at various flying conditions. These systems include additional components as control surfaces to enhance flight performance, leading to an increase in deadweight accompanied by high maintenance costs. This paper presents an improved blowing and suction system that will improve the performance as well as enable a reduction in weight of wing by eliminating the need for additional components. In this present work to demonstrate IBSS, a NACA 6321 flat bottom aerofoil is chosen and the results are compared with the baseline aerofoil.

\section{IMPROVED BLOWING AND SUCTION SYSTEM (IBSS)}

In the proposed Improved Blowing and Suction System (IBSS), the injection and suction holes are of the same area and the injection slot is located at the separation point just below the maximum thickness point and the suction slot is 
located at the circulation creating a point that is close to the trailing edge. The IBSS consists of two components: (1) a regular wing without slats and Flaps, (2) a pump connected to an injection and suction system. The wing produces the major portion of lift due to the airflow over it due to the pressure difference between the top and the bottom side of the wing. The velocity difference in the airflow over the wing essentially generates this pressure difference that produces lift. The pump along with the injection and suction system creates an additional flow that is injected into the flow stream over the aerofoil exactly at the point where the flow separation takes place. The suction slot is located where the circulation plays a major role in the boundary layer separation. The pump injects the same mass of air sucked from the trailing edge that is estimated based on velocity prevailing at the flow separation point. The distance between the injection and suction slot is smaller in the case of IBSS which enables a reduction in the secondary flow losses over the aerofoil. IBSS operated by an active pump injects the additional flow without disturbing the main flow.

The secondary flow is made possible by a micro compressor or a small pump that will work at very high efficiency. Thus by implementing a novel IBSS method secondary control surfaces can be eliminated and increase the aerodynamic performance of the aircraft wing by increasing the stalling angle of attack to a maximum possible value. IBSS shows an enhanced performance of the aircraft wing with less power required for the system and also the losses being minimized. Also, this system can act as a backup system in case of control system failures. Figure 1 shows the schematic representation of the baseline, existing and IBSS aerofoil configurations used in the present study.
Table 1. Aerofoil specifications used in the study

\begin{tabular}{|c|l|c|}
\hline SI. No. & \multicolumn{1}{|c|}{ Details } & Specification \\
\hline 1 & Aerofoil & NACA 6321 \\
\hline 2 & Span $(\mathrm{cm})$ & 30 \\
\hline 3 & Chord $(\mathrm{cm})$ & 30 \\
\hline 4 & Maximum Camber $(\%)$ & 6 \\
\hline 5 & Maximum camber position $(\%)$ & 30 \\
\hline 6 & Thickness $(\%$ of chord) & 21 \\
\hline
\end{tabular}

\section{NUMERICAL ANALYSIS}

The baseline and IBSS aerofoils with the specifications as in Table 1 are modelled for different angle of attack.

The modelled wing is divided into small elements with tetrahedral and prism meshes to obtain optimum results during the numerical analysis. The entire wing model is divided into 1200000 elements to get more accurate results and the mesh is finalized by performing a gridindependent study. The analysis is carried out at a velocity of $35 \mathrm{~m} / \mathrm{sec}$ at a different angle of attacks. Reynolds number used in this analysis is $7.14 \times 10^{5}$. The boundary conditions are provided separately for top aerofoil, bottom aerofoil and IBSS system and depending upon the analysis the boundary conditions are modified. Based on the reported literature, the k-omega method solver is chosen for this numerical analysis which is carried out till convergence. The analysis of the baseline aerofoil is conducted using the regular method, whereas the IBSS analysis is conducted by adding additional mass of flow at the injection location while the same amount is removed from the suction point using a pump. Constant velocity conditions and different angle of attack are used to analyse the aerodynamic parameters and the results are obtained.

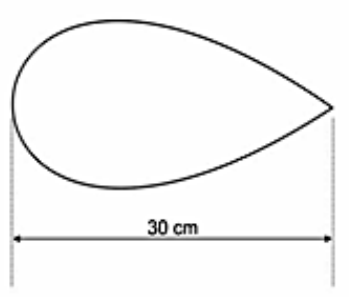

Baseline Aerofoil

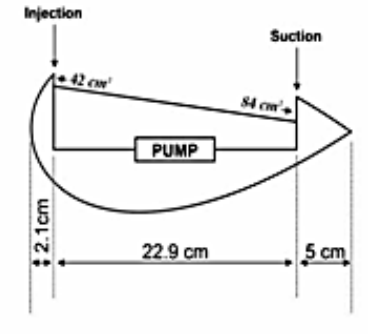

CFJ Aerofoil

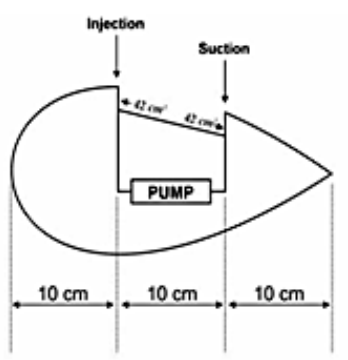

IBSS Aerofoil

Fig. 1. Schematic representation of the base line, CFJ and IBSS aerofoil configurations 


\section{RESULTS}

Numerical analysis is conducted at constant velocity and different angle of attack for baseline, CFJ and IBSS aerofoils. The IBSS model performance is compared with the baseline and existing CFJ method. The IBSS system is also analysed for partial failure conditions and the results are obtained. The analysis is presented under three cases. Case 1: Performance of IBSS vs Baseline aerofoil, Case 2: Performance of IBSS vs Co flow jet aerofoil and Case 3: Performance of IBSS under partial Failure Conditions.

\section{Case 1 - Performance of IBSS and Baseline aerofoil}

The aerodynamic performance of the baseline aerofoil and IBSS is analysed below. Figure 2 shows the velocity distribution over the baseline and IBSS aerofoils. From the velocity distribution as above, it is seen that the boundary layer separation in the baseline aerofoil occurs at an angle of attack of $16^{\circ}$, whereas in the proposed IBSS, the boundary layer separation is suppressed at the same angle of attack. The simulation results are tabulated in Table 2.

From Figure 3 it is seen that the baseline aerofoil undergoes stall at $10^{\circ}$ angle of attack, whereas with the implementation of IBSS, the stall angle is furthered to $16^{\circ}$. It is seen that the IBSS aerofoil performs well at a higher angle of attack compared to baseline aerofoil. Figure 4 shows the comparison of the coefficient of drag produced in the aerofoils at various conditions. The comparison shows that IBSS and Baseline aerofoil produces almost similar drag during various angles of attack. It can be concluded that the

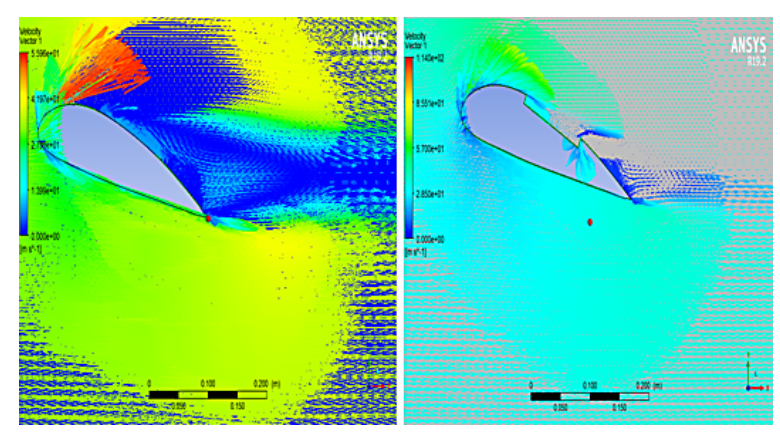

Fig. 2. Velocity distribution over baseline aerofoil and IBSS aerofoil

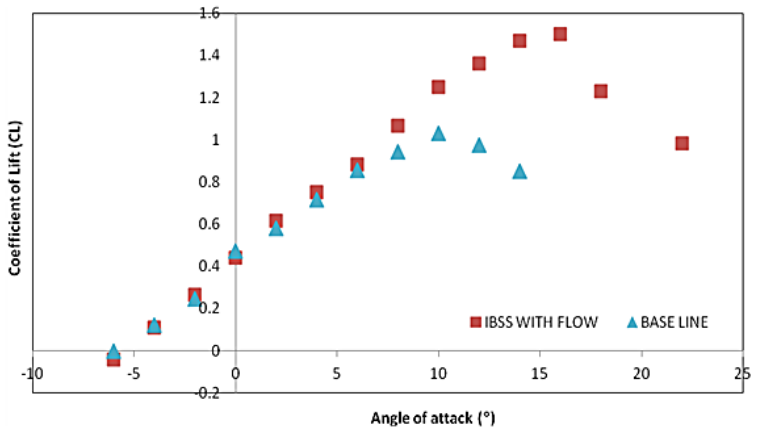

Fig. 3. Variation of coefficient of lift of the aerofoils at different angle of attack

Table 2. Simulation study of baseline and IBSS aerofoils

\begin{tabular}{|c|c|c|c|c|c|c|c|c|c|c|c|c|c|c|}
\hline \multirow{2}{*}{$\begin{array}{c}\text { Angle of } \\
\text { attack } \\
\left(^{\circ}\right)\end{array}$} & \multicolumn{7}{|c|}{ IBSS AEROFOIL } & \multicolumn{7}{|c|}{ BASELINE AEROFOIL } \\
\hline & $C_{L}$ & L & $C_{D}$ & D & L/D & $C_{L} / C_{D}$ & $\mathrm{C}_{\mathrm{m}}$ & $C_{L}$ & L & $C_{D}$ & D & L/D & $\mathrm{C}_{\mathrm{L}} / \mathrm{C}_{\mathrm{D}}$ & $\mathrm{C}_{\mathrm{m}}$ \\
\hline-6 & -0.04 & -2.96 & 0.03 & 1.78 & -1.66 & -1.66 & 0.09 & 0.00 & -0.06 & 0.03 & 1.96 & -0.03 & -0.03 & 0.10 \\
\hline-4 & 0.11 & 7.50 & 0.02 & 1.66 & 4.52 & 4.52 & 0.11 & 0.12 & 8.28 & 0.02 & 1.66 & 4.98 & 4.98 & 0.09 \\
\hline-2 & 0.27 & 17.96 & 0.02 & 1.53 & 11.72 & 11.72 & 0.14 & 0.25 & 16.61 & 0.02 & 1.36 & 12.18 & 12.18 & 0.07 \\
\hline 0 & 0.44 & 29.81 & 0.03 & 1.73 & 17.21 & 17.21 & 0.18 & 0.47 & 31.81 & 0.02 & 1.28 & 24.79 & 24.79 & 0.07 \\
\hline 2 & 0.62 & 41.66 & 0.03 & 1.93 & 21.57 & 21.57 & 0.22 & 0.58 & 39.29 & 0.02 & 1.60 & 24.55 & 24.55 & 0.06 \\
\hline 4 & 0.75 & 50.61 & 0.04 & 2.52 & 20.09 & 20.09 & 0.14 & 0.72 & 48.45 & 0.03 & 2.08 & 23.26 & 23.26 & 0.16 \\
\hline 6 & 0.88 & 59.56 & 0.05 & 3.11 & 19.17 & 19.17 & 0.05 & 0.85 & 57.62 & 0.04 & 2.57 & 22.45 & 22.45 & 0.26 \\
\hline 8 & 1.07 & 71.98 & 0.05 & 3.65 & 19.74 & 19.74 & 0.04 & 0.94 & 63.59 & 0.05 & 3.44 & 18.46 & 18.46 & 0.28 \\
\hline 10 & 1.25 & 84.41 & 0.06 & 4.19 & 20.16 & 20.16 & 0.02 & 1.03 & 69.55 & 0.06 & 4.32 & 16.09 & 16.09 & 0.30 \\
\hline 12 & 1.36 & 91.87 & 0.08 & 5.13 & 17.90 & 17.90 & 0.03 & 0.98 & 65.91 & 0.10 & 6.48 & 10.17 & 10.17 & 0.04 \\
\hline 14 & 1.47 & 99.33 & 0.09 & 6.08 & 16.34 & 16.34 & 0.05 & 0.85 & 57.48 & 0.14 & 9.52 & 6.04 & 6.04 & 0.26 \\
\hline 16 & 1.50 & 101.43 & 0.11 & 7.23 & 14.04 & 14.04 & 0.42 & - & - & - & - & - & - & - \\
\hline 18 & 1.23 & 83.06 & 0.13 & 8.51 & 9.76 & 9.76 & 0.04 & - & - & - & - & - & - & - \\
\hline 22 & 0.98 & 66.31 & 0.30 & 20.39 & 3.25 & 3.25 & 0.50 & - & - & - & - & - & - & - \\
\hline
\end{tabular}




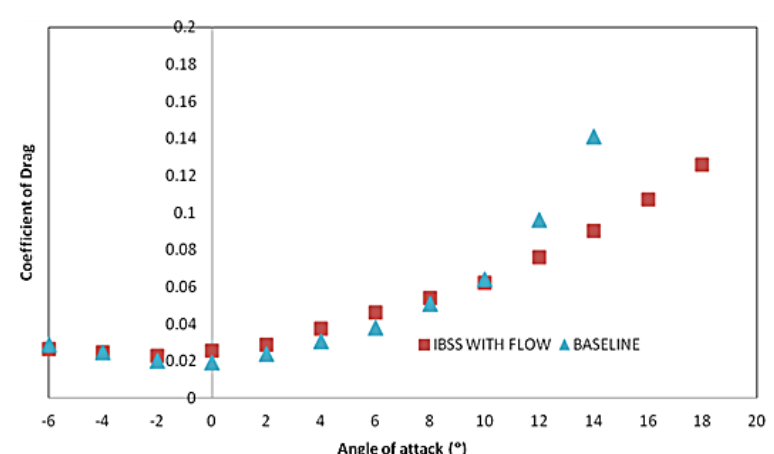

Fig. 4. Variation of coefficient of drag of the aerofoils at different angle of attack

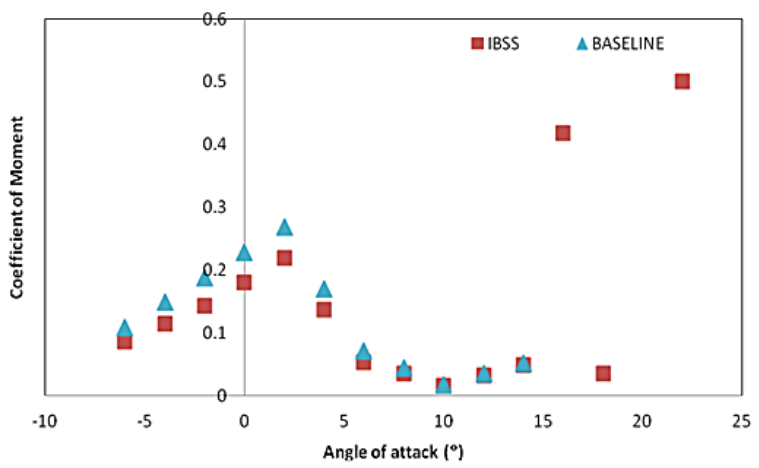

Fig. 5. Comparison of coefficient of moment for IBSS and baseline aerofoils

IBSS method increases the lift without affecting the drag value, thus increasing the aerodynamic efficiency of the wing.

The comparison of $C L$ and $C D$ values of both the aerofoils are shown in Figures 6 and 7. Figure 5 provides the comparison of coefficient of moment values at a different angle of attack for the baseline and IBSS aerofoils. From the graph, it is evident that the IBSS produces more moment at low angle of attack and low moment force at high angle of attacks. Figure 6 shows the comparison of $\mathrm{CL} / \mathrm{CD}$ ratio, an indicator of the efficiency of the aerofoil, at a different angle of attack for both baseline and IBSS aerofoils. It is seen that IBSS method shows improved aerofoil efficiency at a high angle of attack compared to the baseline aerofoil. It is also found that at a lower level angle of attack both the baseline and IBSS aerofoil perform the same. Figure 7 shows the variation of coefficient of lift and coefficient of drag for the baseline and IBSS aerofoils and it is seen that the performance of IBSS is good at a higher angle of attack whereas the same performance as that of the baseline aerofoil at a lower angle of attacks.

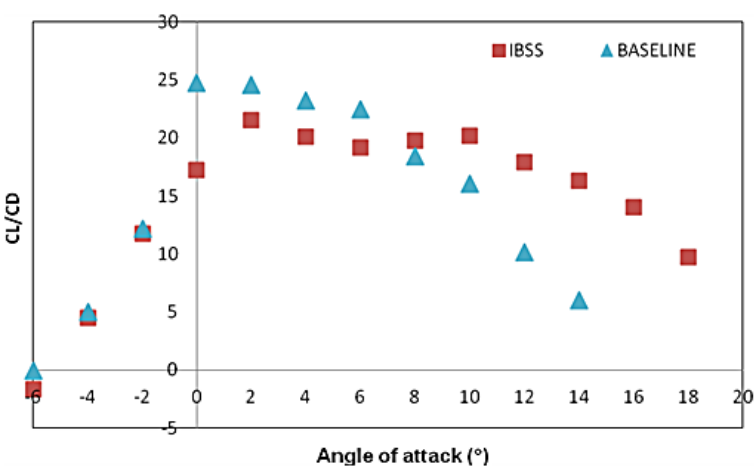

Fig. 6. Comparison of coefficient of lift to drag ratio at different condition

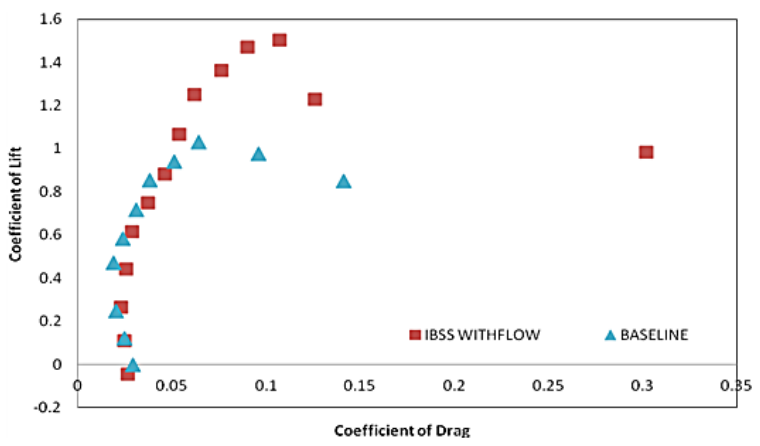

Fig. 7. Comparison of coefficient of lift to drag ratio at different condition

\section{Case 2 - Performance of IBSS and CFJ aerofoil}

The performance of IBSS is compared with the Co flow jet aerofoil and the performance is compared with that of the baseline aerofoil at various conditions. The difference between coflow jet and IBSS aerofoils are the location of the Injection and suction and its areas. The results of the comparison between CFJ and IBSS aerofoils are tabulated in Table 3.

Figure 8 depicts the comparison of the coefficient of lift at the various angle of attack for the different configurations analysed. It can be seen that the baseline aerofoil undergoes stall at a $10^{\circ}$ angle of attack, the CFJ aerofoil gets stalled at $12^{\circ}$ angle of attack and the IBSS aerofoil at $16^{\circ}$. Hence, it is seen that the IBSS method is providing $40 \%$ improvement in stall angle of attack compared to the CFJ aerofoil. Figure 9 shows the comparison of coefficient of drag values of the CFJ and IBSS methods at a different angle of attacks. The CFJ method is seen to create more drag compared to the baseline and IBSS systems. Thus, the IBSS system is seen to produce more 
Table 3. Simulation study of CFJ and IBSS aerofoils

\begin{tabular}{|c|c|c|c|c|c|c|c|c|c|c|c|c|c|c|}
\hline \multirow{2}{*}{$\begin{array}{l}\text { Angle of } \\
\text { attack } \\
\left({ }^{\circ}\right)\end{array}$} & \multicolumn{7}{|c|}{ IBSS AEROFOIL } & \multicolumn{7}{|c|}{ CFJ AEROFOIL } \\
\hline & $\mathrm{C}_{\mathrm{L}}$ & L & $C_{D}$ & D & L/D & $C_{L} / C_{D}$ & $\mathrm{C}_{\mathrm{m}}$ & $C_{L}$ & L & $C_{D}$ & $\mathrm{D}$ & $\mathrm{L} / \mathrm{D}$ & $C_{L} / C_{D}$ & $\mathrm{C}_{\mathrm{m}}$ \\
\hline 0 & 0.62 & 41.66 & 0.03 & 1.93 & 21.57 & 21.57 & 0.22 & 0.26 & 17.62 & 0.05 & 3.65 & 4.83 & 4.83 & 0.13 \\
\hline 2 & 0.75 & 50.61 & 0.04 & 2.52 & 20.09 & 20.09 & 0.14 & 0.37 & 25.26 & 0.08 & 5.06 & 4.99 & 4.99 & 0.16 \\
\hline 4 & 0.88 & 59.56 & 0.05 & 3.11 & 19.17 & 19.17 & 0.05 & 0.49 & 32.89 & 0.10 & 6.48 & 5.07 & 5.07 & 0.20 \\
\hline 6 & 1.07 & 71.98 & 0.05 & 3.65 & 19.74 & 19.74 & 0.04 & 0.71 & 48.15 & 0.14 & 9.32 & 5.17 & 5.17 & 0.27 \\
\hline 8 & 1.25 & 84.41 & 0.06 & 4.19 & 20.16 & 20.16 & 0.02 & 0.96 & 64.64 & 0.15 & 10.40 & 6.22 & 6.22 & 0.38 \\
\hline 10 & 1.36 & 91.87 & 0.08 & 5.13 & 17.90 & 17.90 & 0.03 & 1.20 & 81.14 & 0.17 & 11.41 & 7.11 & 7.11 & 0.50 \\
\hline 12 & 1.47 & 99.33 & 0.09 & 6.08 & 16.34 & 16.34 & 0.05 & 1.69 & 114.12 & 0.18 & 12.22 & 9.34 & 9.34 & 0.63 \\
\hline 14 & 1.50 & 101.43 & 0.11 & 7.23 & 14.04 & 14.04 & 0.42 & 1.40 & 94.20 & 0.21 & 14.38 & 6.55 & 6.55 & 0.61 \\
\hline 16 & 1.23 & 83.06 & 0.13 & 8.51 & 9.76 & 9.76 & 0.04 & 1.10 & 74.28 & 0.30 & 20.33 & 3.65 & 3.65 & 0.52 \\
\hline 18 & 0.44 & 29.81 & 0.03 & 1.73 & 17.21 & 17.21 & 0.18 & - & - & - & - & - & - & - \\
\hline
\end{tabular}

lift at the same time with a reduction in drag compared to its counterparts providing improved performance at high and low angle of attack.

Figure 10 shows the variation of coefficient of moment for the various angle of attacks in the aerofoils compared in this study. It is evident from the above graph that the coefficient of moment in the IBSS method ensures good stability to the aircraft.

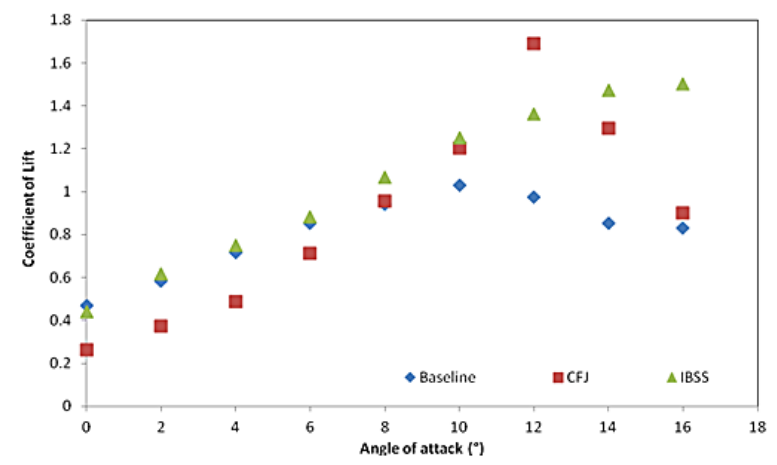

Fig. 8. Comparison of coefficient of lift for CFJ and IBSS aerofoils

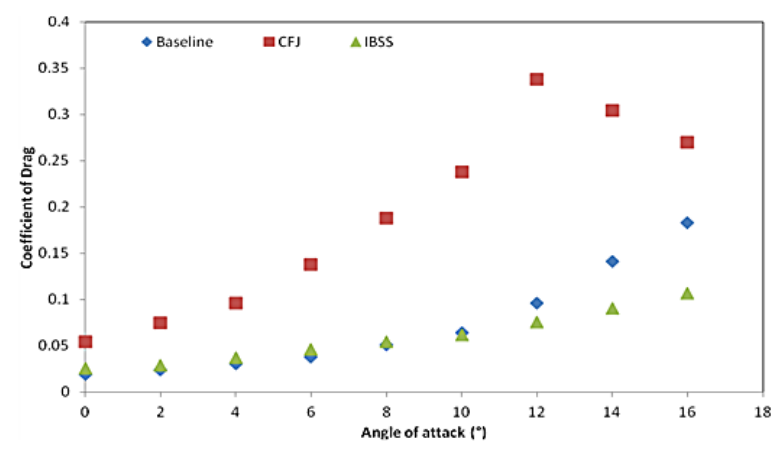

Fig. 9. Comparison of coefficient of drag for CFJ and IBSS aerofoils
However, the CFJ aerofoil is seen to have stability issues due to the boundary layer separation. Figure 11 shows the variation of the coefficient of lift and drag ratios at the various angle of attack. It is seen that the baseline and IBSS aerofoils have more CL/CD values compared to the CFJ aerofoil. It can be concluded that compared to CFJ aerofoil, IBSS aerofoil provides good aerodynamic efficiency. The CFJ aerofoil

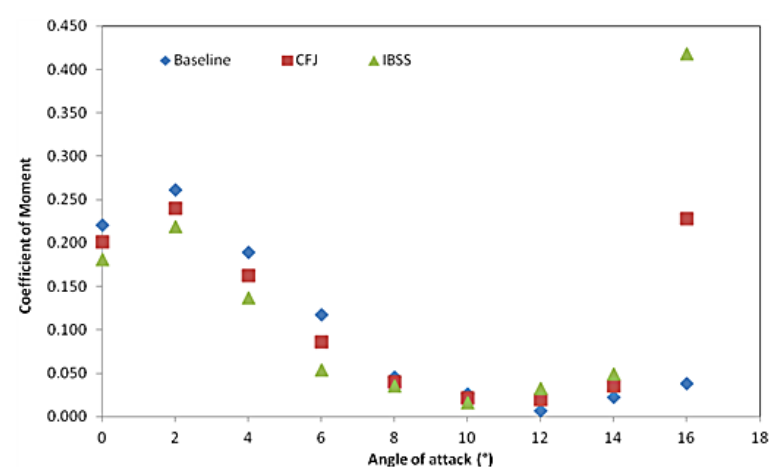

Fig. 10. Comparison of coefficient of moment for CFJ and IBSS aerofoils

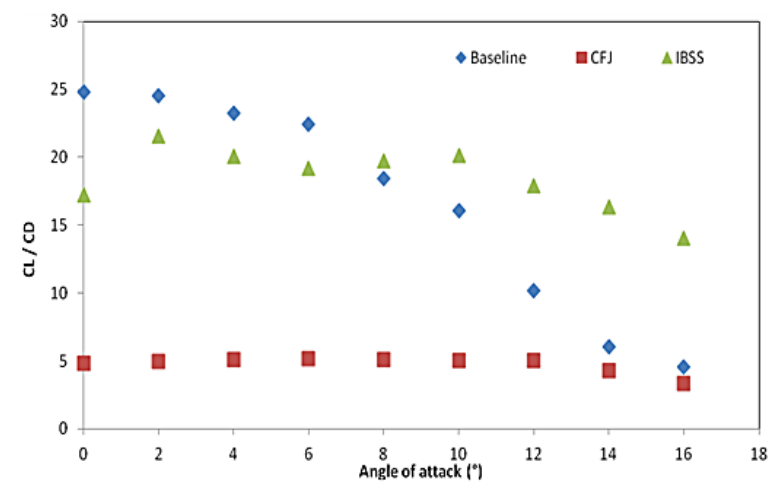

Fig. 11. Comparison of coefficient of lift to drag ratio for CFJ and IBSS aerofoils 
provides slightly better aerodynamic efficiency than the baseline aerofoil at a high angle of attack.

The variation of CL vs $C D$ at various operating conditions for the aerofoils is shown in Figure 12. The drag polar curve for baseline and IBSS aerofoils are quite similar, but in CFJ aerofoil drag polar curve varies because of the distance between the injection and suction points, showing a drastic increase in lift and drag. Hence IBSS aerofoils provide better performance in all conditions. Figure 13 shows the comparison of the L/D ratio of the baseline and IBSS aerofoils. The value trends in the case of both baseline and IBSS methods match whereas the $\mathrm{L} / \mathrm{D}$ value for CFJ aerofoil is seen to have reduced considerably due to the shape modification of the aerofoil. Hence it can be concluded that both CFJ and IBSS methods can be used to increase the coefficient of lift value with IBSS aerofoils performing better than the CFJ aerofoils.

\section{Case 3 - Performance of IBSS under partial Failure Conditions}

Having seen that the IBSS performance is far better than the baseline and CFJ aerofoils, the performance of IBSS aerofoil under partial failure conditions like a suction failure or injection failure is analysed. The results of the performance of IBSS aerofoil under partial failure conditions are discussed in Table 4. From the velocity distribution shown in Figure 14, it is seen that the boundary layer separation and wake region over an aerofoil at partial working conditions are clearly defined. In the IBSS injection method CL value is reduced due to more wake region formation and

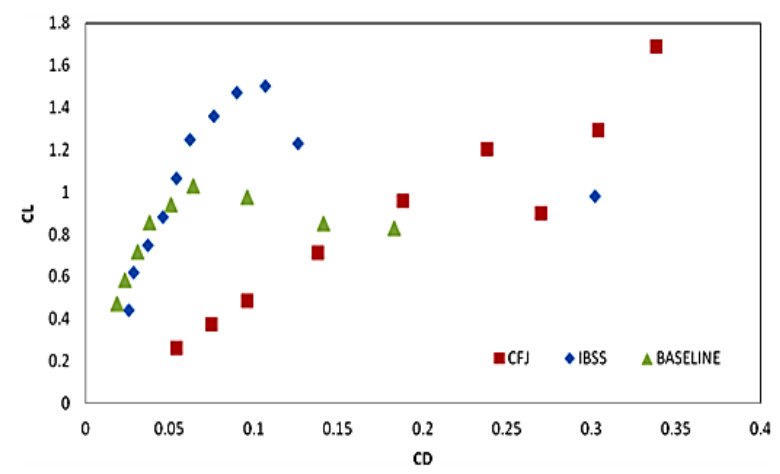

Fig. 12. Comparison of coefficient of lift and drag coefficients for CFJ and IBSS aerofoils

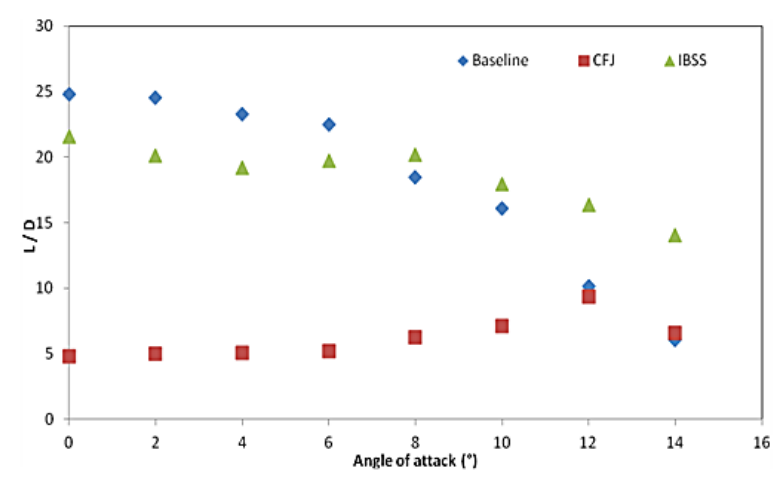

Fig. 13. Lift to drag comparison for various angle of attack for CFJ and IBSS aerofoils

Table 4. Simulation of IBSS aerofoils at partial failure conditions

\begin{tabular}{|c|c|c|c|c|c|c|c|c|c|c|c|c|c|c|}
\hline \multirow{2}{*}{$\begin{array}{l}\text { Angle of } \\
\text { attack } \\
\left({ }^{\circ}\right)\end{array}$} & \multicolumn{7}{|c|}{ IBSS AEROFOIL WITH SUCTION FAILURE } & \multicolumn{7}{|c|}{ IBSS AEROFOIL WITH INJECTION FAILURE } \\
\hline & $C_{L}$ & L & $C_{D}$ & D & L/D & $\mathrm{C}_{\mathrm{L}} / \mathrm{C}_{\mathrm{D}}$ & $\mathrm{C}_{\mathrm{m}}$ & $C_{L}$ & L & $C_{D}$ & $\mathrm{D}$ & L/D & $\mathrm{C}_{\mathrm{L}} / \mathrm{C}_{\mathrm{D}}$ & $\mathrm{C}_{\mathrm{m}}$ \\
\hline-6 & -0.08 & -5.47 & 0.13 & 8.78 & -0.62 & -0.62 & -0.19 & 0.13 & 8.85 & 0.04 & 2.53 & 3.49 & 3.49 & 0.14 \\
\hline-4 & -0.31 & -20.66 & 0.10 & 6.90 & -2.99 & -2.99 & -0.17 & 0.35 & 23.37 & 0.05 & 3.56 & 6.56 & 6.56 & 0.19 \\
\hline-2 & -0.53 & -35.86 & 0.07 & 5.02 & -7.14 & -7.14 & -0.14 & 0.56 & 37.89 & 0.07 & 4.59 & 8.25 & 8.25 & 0.24 \\
\hline 0 & -0.33 & -22.08 & 0.05 & 3.70 & -5.97 & -5.97 & -0.09 & 0.78 & 52.84 & 0.08 & 5.45 & 9.70 & 9.70 & 0.30 \\
\hline 2 & -0.12 & -8.31 & 0.04 & 2.37 & -3.50 & -3.50 & -0.03 & 1.00 & 67.80 & 0.09 & 6.30 & 10.7 & 10.76 & 0.35 \\
\hline 4 & 0.08 & 5.37 & 0.03 & 1.85 & 2.90 & 2.90 & -0.02 & 1.22 & 82.18 & 0.10 & 6.66 & 12.34 & 12.34 & 0.23 \\
\hline 6 & 0.28 & 19.04 & 0.02 & 1.33 & 14.31 & 14.31 & 0.00 & 1.43 & 96.57 & 0.10 & 7.02 & 13.75 & 13.75 & 0.11 \\
\hline 8 & 0.48 & 32.11 & 0.02 & 1.54 & 20.81 & 20.81 & 0.00 & 1.67 & 112.94 & 0.11 & 7.16 & 15.78 & 15.78 & 0.11 \\
\hline 10 & 0.67 & 45.18 & 0.03 & 1.76 & 25.73 & 25.73 & 0.00 & 1.92 & 129.32 & 0.11 & 7.29 & 17.73 & 17.73 & 0.12 \\
\hline 12 & 0.85 & 57.37 & 0.04 & 2.51 & 22.87 & 22.87 & 0.00 & 2.11 & 142.32 & 0.12 & 7.90 & 18.01 & 18.01 & 0.12 \\
\hline 14 & 1.03 & 69.55 & 0.05 & 3.26 & 21.33 & 21.33 & 0.01 & 2.30 & 155.31 & 0.13 & 8.51 & 18.25 & 18.25 & 0.13 \\
\hline 16 & 1.19 & 80.36 & 0.07 & 4.50 & 17.87 & 17.87 & 0.31 & 2.50 & 168.82 & 0.14 & 9.39 & 17.99 & 17.99 & 0.76 \\
\hline 18 & 1.32 & 89.14 & 0.10 & 6.42 & 13.89 & 13.89 & 0.01 & 2.71 & 183.00 & 0.16 & 10.80 & 16.94 & 16.94 & 0.15 \\
\hline 22 & 0.98 & 66.18 & 0.25 & 8.65 & 3.94 & 3.94 & 0.40 & 3.10 & 209.34 & 0.37 & 24.72 & 8.47 & 8.47 & 1.08 \\
\hline
\end{tabular}



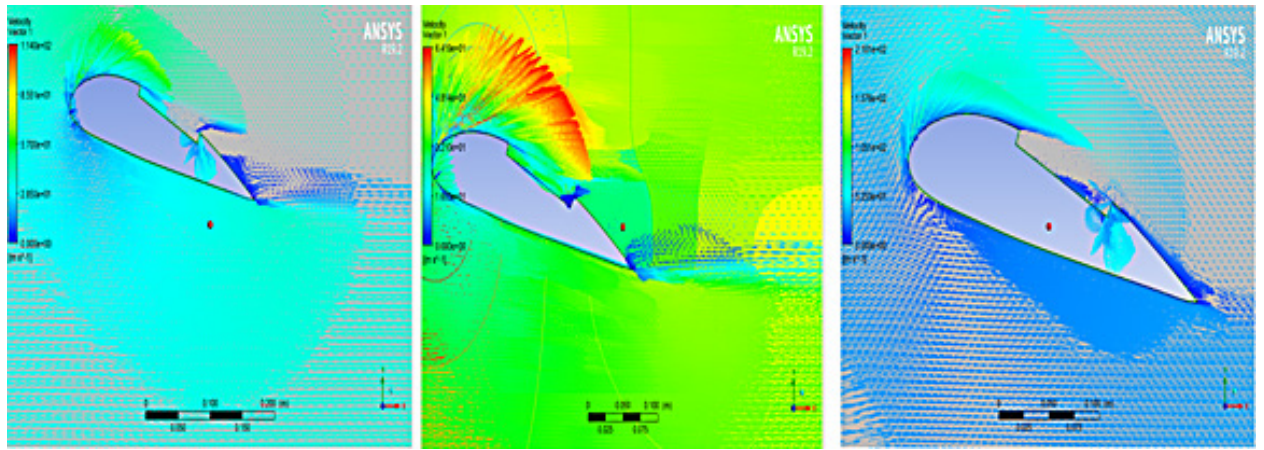

Fig. 14. Schematic representation of velocity distribution at partial failure conditions

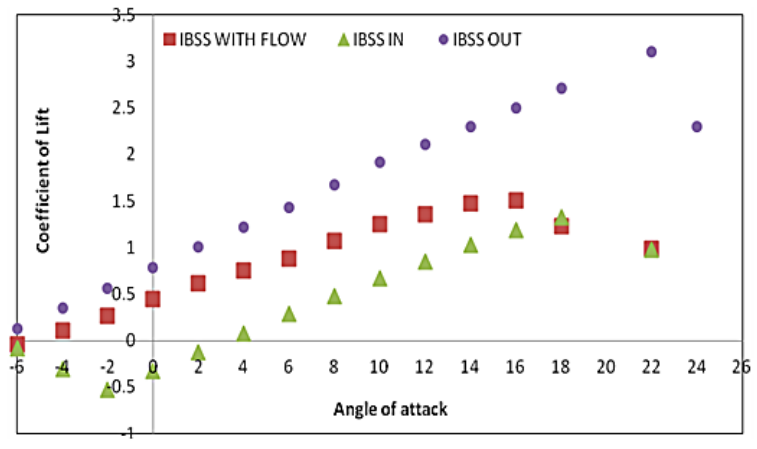

Fig. 15. Comparison of coefficient of lift versus angle of attack at partial working condition

the IBSS suction method is seen to increase the coefficient of lift due of less wake region. The comparison of CL and CD values of IBSS at partial failure conditions are shown in Figures 18 and 19. The IBSS is analysed for two conditions namely injection only and Suction only and final results are compared with IBSS regular working conditions. From Figure 15 it is seen that the IBSS aerofoil can attain a maximum angle of attack of $16^{\circ}$, whereas with the IBSS injection system only working, the stalling angle of attack is $18^{\circ}$ which indicates a further improvement of $12.5 \%$, however, the CL value is reduced marginally due to the presence of wake region close to the trailing edge. IBSS suction-only system shows that the stalling angle of attack is furthered to $22^{\circ}$ which is $37.5 \%$ higher because the wake region reduced close to the trailing edge.

Figure 16 shows the comparison of the coefficient of drag produced in the aerofoils at various conditions. The comparison shows that IBSS injection and IBSS is producing less amount of drag compared to the IBSS suction condition. In IBSS and IBSS injection condition, additional flow is introduced into an aerofoil without disturbing the main flow which decreases the drag during various angles of attack. In IBSS suction condition, drag is increased because of no additional flow over the aerofoil. Figure 17 provides the comparison of coefficient of moment values at a different angle of attack for the IBSS aerofoils partial failure conditions. From the graph, it is evident that the IBSS produces moderate moment at various angles of attack. In IBSS injection the

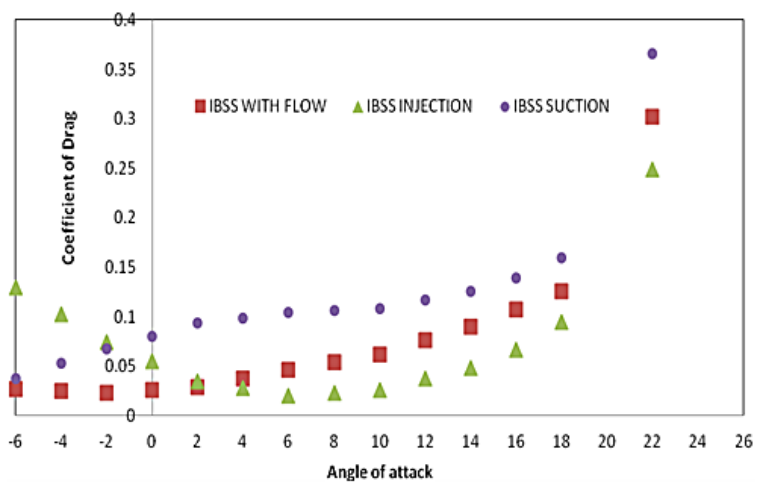

Fig. 16. Comparison of coefficient of drag versus angle of attack at partial working condition

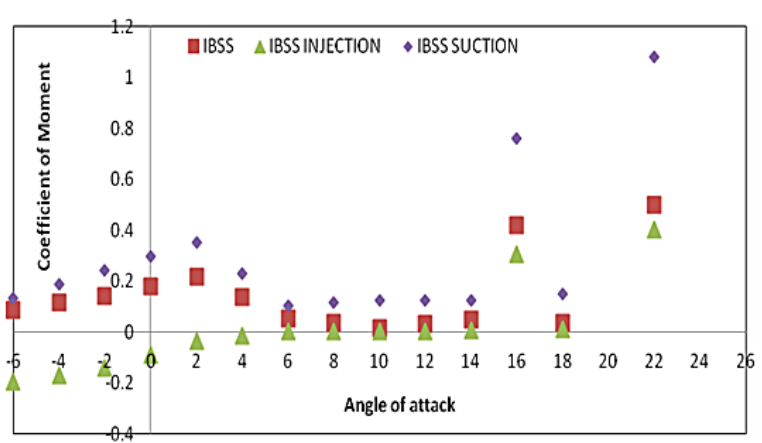

Fig. 17. Comparison of coefficient of moment versus angle of attack at partial working condition 


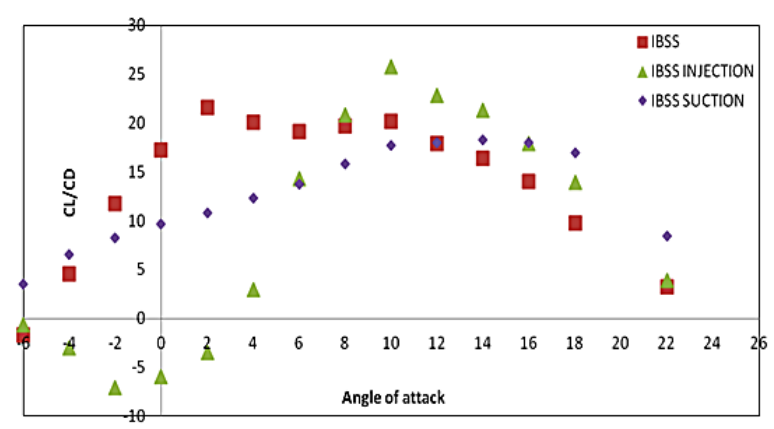

Fig. 18. Comparison of coefficient of lift to drag curve at various angle of attack at partial working condition

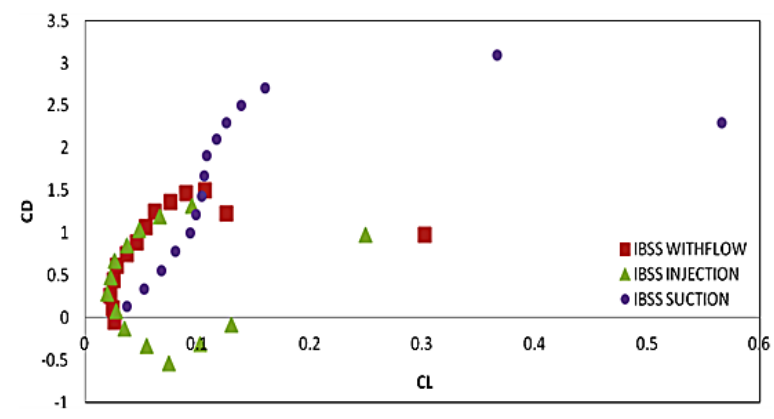

Fig. 19. Coefficient of lift vs coefficient of drag curve at partial working condition

moment produced is less and during IBSS suction is more at various angles of attack compared to IBSS normal working condition because of boundary layer suppression.

Figure 18 shows the variation of the coefficient of lifts and drag ratios at various angles of attack. In partial failure conditions, IBSS injection system is providing good aerodynamic efficiency at a high angle of attack and the IBSS Suction system is providing close to the IBSS system which is seen to enhance the flight performance of the aircraft. Figure 19 shows the variation of coefficient of lift and coefficient of drag for the IBSS and IBSS partial failure conditions aerofoils. The drag polar curve shows that the performance of IBSS and IBSS injection systems are similar. But the performance of the IBSS Suction system shows a high correlation because of suction taking place at the circulation point and without additional flow.

Figure 20 shows the comparison of the L/D ratio of the IBSS and its partial failure conditions. This curve shows that the aerodynamics performance of the IBSS injection system is close to that of the IBSS system due to additional flow

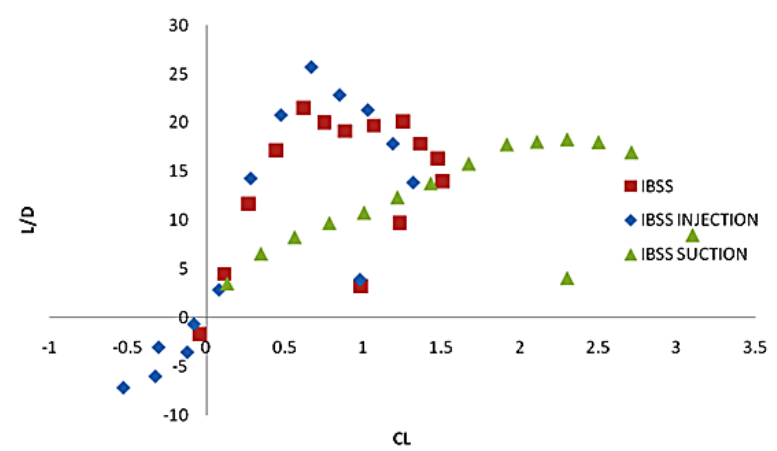

Fig. 20. Lift to drag ratio curve to coefficient of lift curve

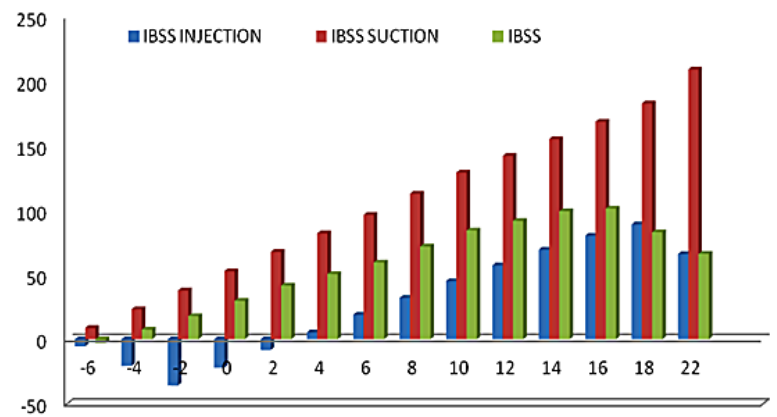

Fig. 21. Comparison of total lift generated

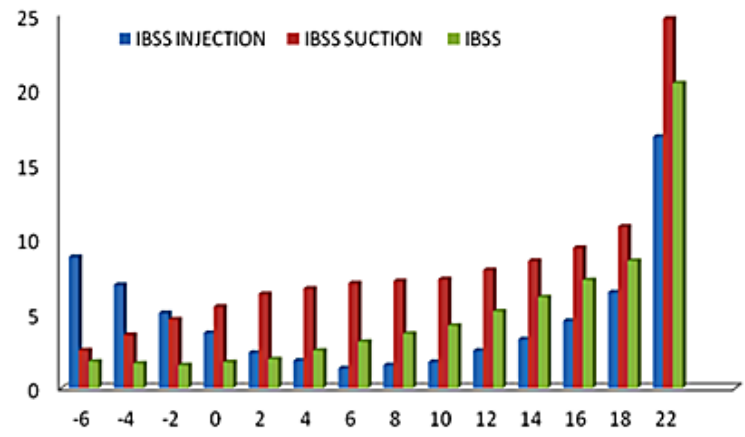

Fig. 22. Comparison of total drag generated

introduced over the aerofoil. In the IBSS Suction system, the aerodynamic performances are reduced to some amount instead of that CL values are greatly improved as compared to IBSS and IBSS Injection systems because of the reduced wake region. Hence it is seen that the IBSS system also works effectively and safely even during a partial failure condition. Figure 21 show comparison of total lift at the various angle of attack at different conditions like IBSS injection, IBSS suction and IBSS operating conditions. It is proved that the IBSS injection method slowly 
enhances the lift after the positive angle of attack of an aerofoil. In IBSS suction condition, the lift is enhanced right from the negative angle of attack to higher angle of attack conditions. IBSS method is producing the average and correct amount of lift at different velocities. So IBSS method at full and partial operating conditions is producing a particular amount of lift and improve the aerodynamics performance depending on the operating conditions. Figure 22 displays that the total drag generated by an aerofoil at different operating conditions like IBSS and its partial failure conditions. In the IBSS injection method, it is seen that the maximum amount of drag at negative and high angle of attack is produced because of no suction of flow close to the trailing edge. IBSS suction method is producing more amount of drag at all angle of attack due to lack of additional flow over the aerofoil. IBSS method is generating a gradual increase of drag from low to the high angle of attack due to the additional flow properly circulated over an aerofoil.

\section{CONCLUSIONS}

The aerodynamic performance of a novel aerofoil modified with improved blowing and suction system (IBSS) is analysed and the performance is compared with that of a baseline aerofoil and CFJ modified aerofoil. The IBSS aerofoil is also analysed for partial failure conditions and the following conclusions are obtained.

With minimum modifications in the existing flow control mechanisms in practice, IBSS aerofoil effectively delays the boundary layer separation over the aerofoil. IBSS controls the boundary layer separation effectively and thus improves the aerodynamic efficiency of the wing which makes it best suited for all kinds of low-speed aircrafts, thereby addressing a major problem existing in today's aircraft. The proposed IBSS wing can operate efficiently at both low as well as the high angle of attacks. Also, in case of any failure in the injection or suction system of the IBSS, the normal performance of the wing is not affected, rather improved. Even in case of complete failure in the suction and injection systems, the performance will gradually deteriorate providing enough time for the pilots to take appropriate rescue actions. IBSS method is seen to improve the stalling angle of attack up to $60 \%$ without increasing the drag compared to the baseline aerofoil due to the boundary layer suppression over the aircraft wing. IBSS method is seen to increase the stalling angle of attack up to $40 \%$ without increasing the drag as compared to the existing co-flow jet mechanism because of the modified injection location. IBSS method is found to work effectively during partial failure conditions. It is proved that stalling angle of attack is improved by $12.5 \%$ during the injection only condition, compared to normal IBSS operating condition. But CL value is decreased to a considerable amount due to ineffective working of the suction portion. In the IBSS suction condition, the stalling angle of attack is improved by $37.5 \%$ compared to the normal IBSS operating condition. In this case, the CL value is increased because of the vacuum region which is located close to the trailing edge. Also, in the IBSS method, the distance between the injection and suction points are less and hence the pumping power required and losses between the injection and suction is also reduced compared to the existing methods. IBSS method is used to keep injection and suction areas constant to achieve good aerodynamic performance over an aerofoil.

\section{REFERENCES}

1. Zha G.C., Paxton C., A novel airfoil circulation augment flow control method using co-flow jet. In: Proc. of the $2^{\text {nd }}$ AIAA Flow Control Conf., 2004, 1-13. https://doi.org/10.2514/6.2004-2208.

2. Zha G.C., Paxton C., Novel flow control method for airfoil performance enhancement using co-flow jet. In: Applications of Circulation Control Technology 2006, 293-314. https://doi.org/10.2514/5.9781600 866838.0293.0314.

3. Lefebvre A., Zha G.C., Co-flow jet airfoil trade study, Part I: Energy consumption and aerodynamic efficiency. In: Proc. of the $32^{\text {nd }}$ AIAA Appl. Aerodyn. Conf. 2014, 1-20. https://doi. org/10.2514/6.2014-2682.

4. Zha G.C., Gao W., Paxton C., Gables C., Numerical simulation of co-flow jet airfoil flows. Proc. of the. $44^{\text {th }}$ AIAA Aerospace Sciences Meeting and Exhibit, 2006, 1-11.

5. Zha G.C., Carroll B.F., Paxton C.D, Conley C.A, Wells A., High-performance airfoil using coflow jet flow control. AIAA Journal, 2007, 45(8), 1-27. https://doi.org/10.2514/1.20926

6. Hossain M.A., Uddin M.N., Islam M.R., Mashud M. Enhancement of aerodynamic properties of an airfoil by co flow jet. American Journal of Engineering Research. 2015, 4(1), 103-112. 
7. Lefebvre A., Dano B., Bartow W.B., Fronzo M.D., Zha G.C. Performance and energy expenditure of coflow jet airfoil with variation of mach number. Journal of Aircraft. 2016, 53(6), 1757-1767. https://doi.org/10.2514/1.c033113.

8. Balaji K., Wessley G.J.J. Experimental investigations on the performance of a modified co-flow jet aerofoil. Indian Journal of Science and Technology, 2020, 13, 1873-1881. 10.17485/IJST/v13i19.85.
9. Lefebvre A., Zha G.C., Trade study of 3D co-flow jet wing for cruise and takeoff/landing performance. In: Proc. of the $54^{\text {th }}$ AIAA Aerosp. Sci. Meet., 2016, 1-29. https://doi.org/10.2514/6.2016-0570.

10. Dano B.P.E., Kirk D., Zha G.C. Experimental investigation of jet mixing mechanism of a co-flow jet airfoil. In: Proc. of the $5^{\text {th }}$ AIAA Flow Control Conference, 2010. https://doi. org/10.2514/6.2010-4421. 